\title{
THE OUTLOOK FOR MATHEMATICS ON THE WEB
}

\author{
BRADFORD D. ALLEN \\ Florida Institute of Technology \\ Department of Mathematical Sciences \\ Melbourne, FL 32901 USA \\ ballen@fit.edu
}

(Received March 2000; Revised August 2000)

Cooperation among major software providers to adopt industry-wide standards and ongoing advances in hardware and software will greatly expand the range and power of mathematics on the Internet. Recent technological developments are changing the web environment so that files with mathematical content will soon be easily loaded, sent, received, and processed. To make this possible, mathematics files will contain both typesetting information on how math notation should appear and structural information on how notation should be interpreted. That is, math files on the web will contain both presentation and semantic information to allow math expressions to be transported, evaluated, tested, simplified, graphed, and even graphically animated. This article discusses some of the technological advances that will empower webbased mathematics by making it interactive and portable. Some background on files is discussed first to explain how solutions to certain technological problems will lead to great improvements in the utilization of mathematics on the web.

Files with mathematics content generated by word processors, equation editors, and computer algebra systems contain particular format and structure specifications. This information, called markup, controls the appearance, and in some instances, the function of elements within documents. To be useful on the Internet however, files must conform to various Internet standards that, to a great extent, have excluded mathematics from the web. Unusual characters, context-sensitivity, and specialized markup have made it difficult to adapt math files to an Internet framework. Though generally invisible to the word processor user, markup for mathematics notation has not been compatible with Internet standards and, for the most part, has restricted math notation on the web to a graphic-image format.

The most popular markup language to control the appearance and function of elements in web documents is Hypertext Markup Language (HTML) (www.w3.org/MarkUp). HTML is easily written and read by people and machines, however, HTML is limited by the extent to which the appearance and function of elements may be specified. Though the appearance of a document on the web cannot be defined explicitly, HTML appearance formatting defines attributes such as font style and size. Function formatting, on the other hand, defines the role particular elements of text, data, and symbols play. For example, a web document might contain a string that is formatted as bold and as a title (e.g. $<$ TITLE $><$ B $>$ Math 
on the Web $</$ TITLE $></ B>$ ). The appearance of the title would depend on the Internet browser but the title element itself would always be treated as a title.

Word processors and equation editors have their own markup formatting which makes sharing files among them difficult. However, these files are becoming more portable over networks and across platforms. Microsoft's Office Suite (www.microsoft.com/office/order/suitegde.htm) which includes Word and Excel can now create and interpret HTML web files. This has been good news for text file users but has not helped those wishing to use mathematics on the web. The problem has been that the range of HTML file content (and in particular, mathematical content) is restricted by a fixed set of HTML attribute formats that cannot be extended. What is lacking in HTML markup, and in web software in general, is the ability to specify context-sensitive meanings to mathematical elements. What is needed then, are web technologies that can create and interpret semantic-information-enhanced mathematical notation. With a universally accepted markup formatting language, the interface between math symbols and web browsers would be automatic.

The first step to meet these goals is industry-wide acceptance and adoption of a single standard for representing the wide range of characters and symbols used in mathematics. One such development toward this end is Unicode (www.unicode.org), an international computer standard for representing characters and symbols. In contrast to the American Standard Code for Information Interchange (ASCII), which uses one byte to represent 128 characters, Unicode uses two bytes per character. With two bytes, Unicode can represent all the mathematical, scientific, alphabetic, and numeric symbols commonly used worldwide. Unicode is gaining support from software makers and is presently included in Windows 2000 (www.microsoft.com/ windows2000) and Java technology (http://java.sun.com/).

On the markup language front, many problems associated with HTML are addressed in a recently introduced markup language called XML or Extensible Markup Language (www.xml.com, http://xml.org). XML and HTML are similar as both were derived from the internationally used Standard Generalized Markup Language (SGML)(www.oasis-open.org/ cover). For those wishing to use web-based mathematics however, the important difference between XML and HTML is that XML provides both appearance and structure information for elements of text, data, and symbols. That is, XML not only defines how text, data, and symbols appear in a web document but XML also controls and even expands the roles elements play within a document. This later feature allows similar elements in different contexts to have different meanings within a document. For example, by specifying context-sensitive presentation and semantic information, the superscript " -1 " in a math document would be identified as a part of the function name in $f^{-1}$ and identified as an exponent of a variable in $x^{-1}$.

When semantic information is written into code specifying context-specific roles for a wide range of elements, that XML code may be used as a template for a markup language dialect. In other words, new markup languages may be created using XML's ability to define and extend meanings for elements with respect to context. Thus, specific vernaculars or dialects of XML may be created for various scientific disciplines such as chemistry, biology, and mathematics (www.amsci.org/amsci/issues/ Comsci98/compsci1998-09.html).

The XML dialect of interest to mathematicians is MathML (www.w3.org/Math), a mathematical markup language created by the World Wide Web Consortium Math Working Group (www.w3.org). Unlike the popular TeX and LaTeX typesetting 
systems (www.pctex.com) which currently provide only presentation information, XML based MathML provides both presentation and semantic information for web mathematics. As such, MathML allows mathematical text to be communicated and processed across various computer platforms and applications. With MathML, the potential benefits for web math are great. An equation could be copied directly from a web document and pasted into a computer algebra system such as Matlab (www.mathworks.com), Maple (www.maplesoft.com), or Mathematica (www.mathematica.com). The equation could be simplified, evaluated, tested, graphed, or graphically animated. Lengthy computations could be performed on a fast server computer. For some applications, computations could be done in parallel over a network of computers using Mathematica's Parallel Computing Toolkit (www.mathematica.com). When MathML documents are printed, equations would have properly formatted notation and be scaled in size to conform to the rest of the document. Further, the entire web could be searched for specific MathML equations. In the more distant future, MathML mathematics will be machine read to the visually impaired directly from the web (www.amsci.org/amsci/issues/Comsci98/ compsci1998-09.html).

Industry support for MathML is gaining though there are still many software components that are not XML compatible. While equation editor and word processor products such as MathType (www.mathtype.com) and FrameMaker (www.adobe.com/products/framemaker) will translate math notation into MathML format, XML, and thus MathML, cannot presently be translated from the web by the popular web browsers. However, several venders have software that will read MathML files from the web (www.w3.org/Math). Most important for mathematics on the web, though, is that the two major browser software producers, Microsoft and AOL's Netscape (www.netscape.com), have recently agreed to support MathML in future browser software.

While the math and science community must wait before web mathematics can be used with the power and flexibility offered by MathML, there are several ways mathematics may be used today in web documents. First, mathematics may be written (in limited ways) with Microsoft's Word or Powerpoint equation editors. The resulting files may then be uploaded to a web server using FTP commands (http://cws.internet.com/32ftp.html\#ws-ftp) or with shareware software such as WSFTP (www.imaginarylandscape.com/helpweb/ftp/ftptop.html). If the file is to support or supplement a course in mathematics, HTML based course construction programs such as Web Course in a Box (http://www.madduck.com), WebCT (www.webct.com), or Blackboard (www.blackboard.com) are available. These packages will not only upload math files but will assist in creating entire online math courses. The HTML files generated by these web course programs may be viewed from the web with most browsers.

Second, mathematics may be written using HTML. Though limited in scope, HTML allows superscripts, subscripts, bold, italic, underline, and bar characters. Furthermore, gif or jpg graphic images generated by an equation editor may be placed throughout an HTML file. Once uploaded onto a server, these files may be viewed with free downloaded software designed to read Microsoft files.

Third, a word processor with equation editing capability that can save documents as HTML code interlaced with math graphic images may be used to write and translate mathematical text for the web. For example, Mathtype, FrameMaker, TeX, LaTeX, AMS-TeX, and AMS-LaTeX will convert files into HTML code with math 
graphic images.

Fourth, Matlab, Maple, or Mathematica may be used to create math text, graphs, and even animations. The files may be uploaded and then shared. Anyone downloading these files would just have to open the files with the same computer algebra system that created the files.

The mathematical environment of the web will feature platform-independent expressions that lend themselves to numeric, symbolic, and graphical computations. Web mathematics will move from the confines of the graphic image to become easily accessed, imported, exported, and analytically manipulated. With these possibilities coming into sight, and many more possibilities still open, the outlook is bright for mathematics on the web. 


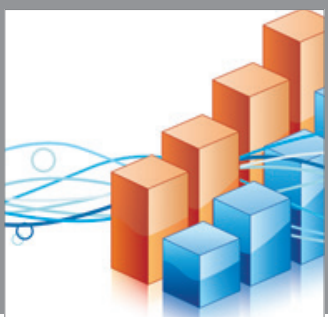

Advances in

Operations Research

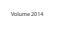

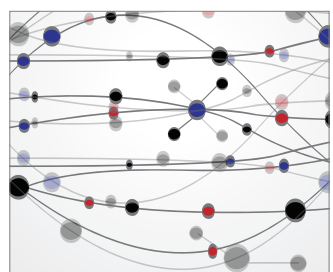

\section{The Scientific} World Journal
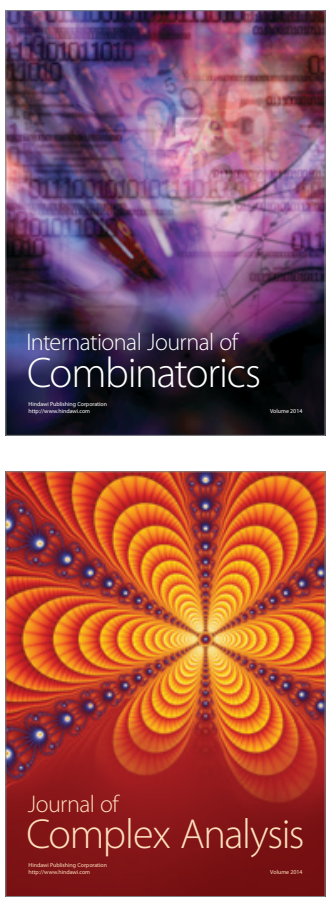

International Journal of

Mathematics and

Mathematical

Sciences
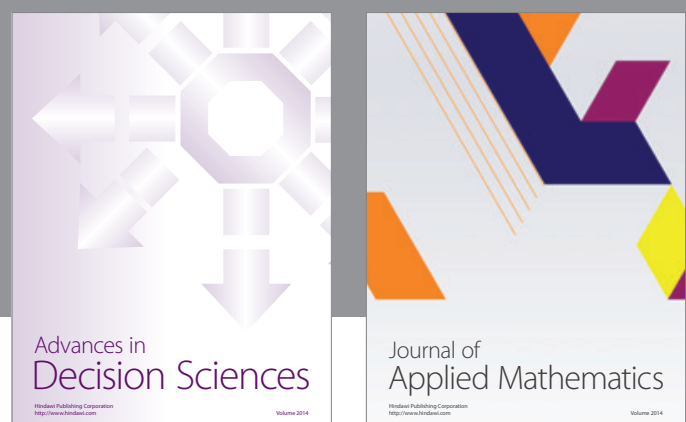

Journal of

Applied Mathematics
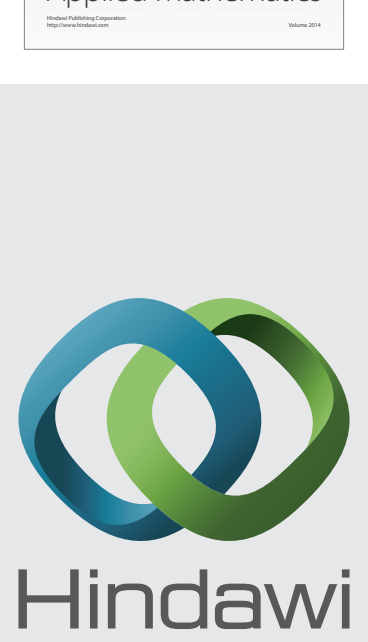

Submit your manuscripts at http://www.hindawi.com
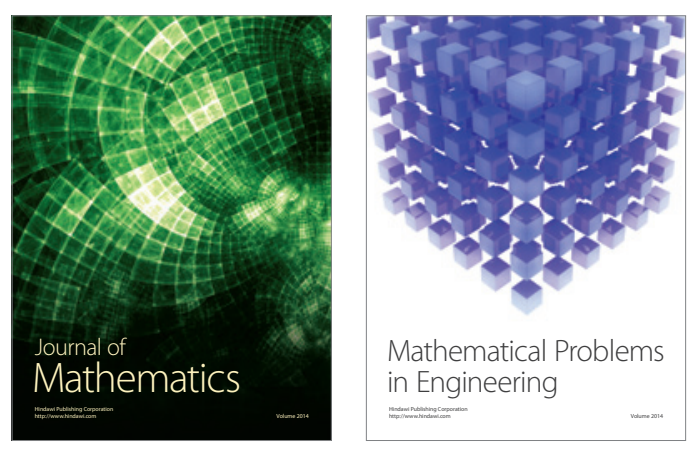

Mathematical Problems in Engineering
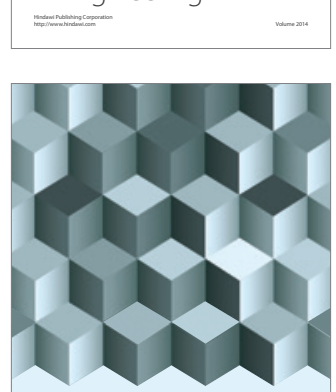

Journal of

Function Spaces
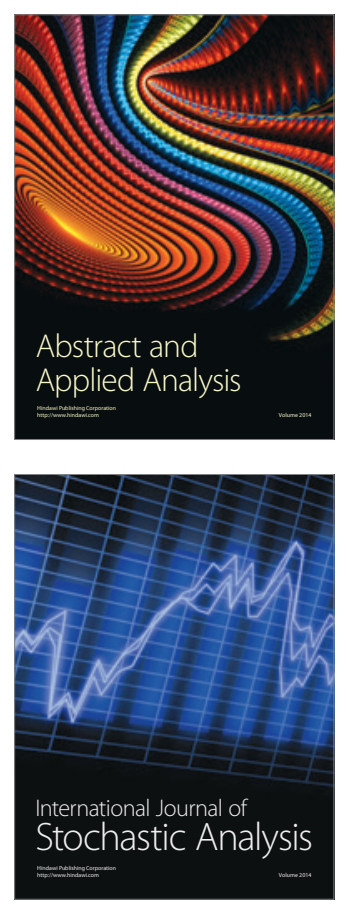

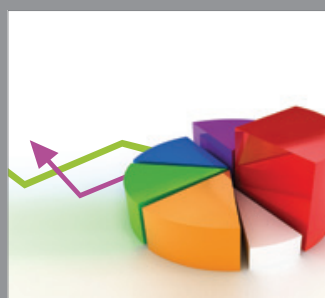

ournal of

Probability and Statistics

Promensencen
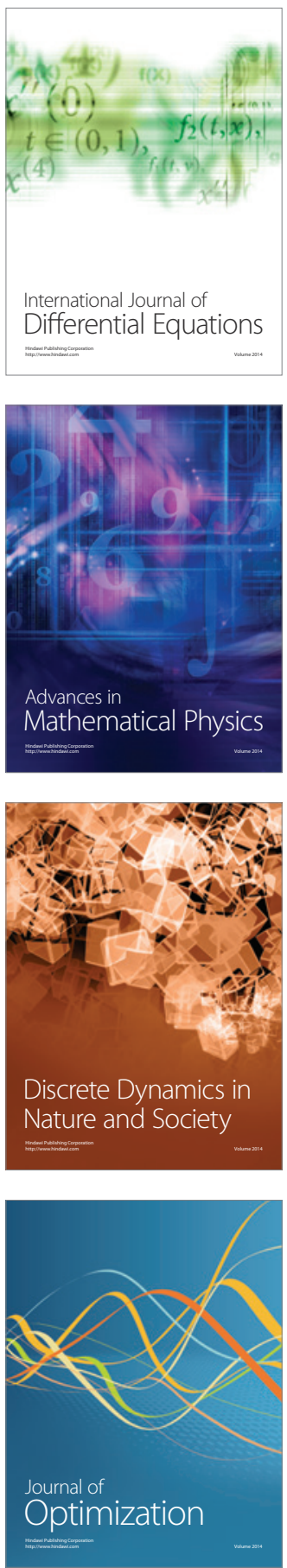